\title{
Characterization of Polymorphic Chloroplast Microsatellites in Prunus Species and Maternal Lineages in Peach Genotypes
}

\author{
Chunxian Chen ${ }^{1}$ and William R. Okie \\ U.S. Department of Agriculture, Agricultural Research Service, Southeastern Fruit and Tree Nut \\ Research Laboratory, 21 Dunbar Road, Byron, GA 31008
}

\begin{abstract}
Additional Index words. Prunus persica, P. kansuensis, P. mume, simple sequence repeat, maternal inheritance, phylogenetic analysis

Abstract. Several available Prunus chloroplast genomes have not been exploited to develop polymorphic chloroplast microsatellites that could be useful in Prunus phylogenetic analysis and maternal lineage group (MLG) categorization. In this study, using available bioinformatics tools, 80, 75, and 78 microsatellites were identified from the chloroplast genome of $P$. persica (CPpe), P. kansuensis (CPka), and $P$. mume (CPmu), respectively. The genome features and polymorphism status of these microsatellites were characterized. The genomic locations and motif types of most chloroplast microsatellites were conserved in CPpe, CPka, and CPmu. Of the 67 microsatellites with primer sequences and names, 57 were polymorphic for their in silico motif, amplicon lengths, or both among the three genomes. Based on the genotyping data of eight most polymorphic microsatellites, eight unique MLGs were found among the 736 peach materials in a breeding program. Most peach cultivars (111 of 161 genotyped) belong to MLG-1, the Chinese Cling-derived group reflecting the heavy use of this germplasm in early peach development. Forty-one cultivars belong to MLG-2, the European-derived group of peaches. MLG-3 consists of ornamental accessions. MLG-4 and MLG-5 contain only 'Flordaking' and 'Reliance', respectively. MLG-6 to MLG-8 consists of selections derived from $P$. tangutica, $P$. davidiana, and $P$. mira, respectively. These amplicons from the representative material for each MLG were sequenced, revealing additional single nucleotide polymorphisms (SNPs) within the amplicons. With the polymorphism status and amplification reliability validated, these new polymorphic chloroplast microsatellite markers may be useful in Prunus phylogenetic analysis.
\end{abstract}

Peach (Prunus persica) was domesticated several thousand years ago in China and subsequently introduced to Persia, the Mediterranean, the Americas, and elsewhere (Faust and Timon, 1995). Cultivars selected for cool dry climates generally do not perform well in hot humid climates or vice versa. The ability of peach to respond to both human as well as natural selection pressure has produced genotypes adapted to a very wide range of climates and cultural preferences (Aranzana et al., 2003; Okie, 1998). Before the introduction of 'Chinese Cling' from China in the $1850 \mathrm{~s}$, most of the peaches grown in the United States were of European origin (Myers et al., 1989). After the introduction of 'Elberta' (a 'Chinese Cling' seedling) in the 1870s followed by its many descendants, conventional breeding programs, particularly in the last century, became the primary generators of cultivar diversity (Aranzana et al., 2003; Cantin et al., 2010; Okie, 1998; Sitther et al., 2012). Accrued introgression and spontaneous mutations also played a role in peach evolution (Chen et al., 2016). Therefore, genetic knowledge of the maternal

Received for publication 7 Feb. 2017. Accepted for publication 2 May 2017. The research was partly supported by the U.S. Department of Agriculture National Program of Plant Genetic Resources, Genomics and Genetic Improvement (Project number: 6606-21000-004-006) and Georgia Agriculture Commodity Commission for Peaches (Project number: 58-6042-5-002). We thank Bryan Blackburn, Minling Zhang, and Luke Quick for their assistance in maintaining the materials. This article reports the results of research only. Mention of a trademark or proprietary product is solely for the purpose of providing specific information and does not constitute a guarantee or warranty of the product by the USDA and does not imply its approval to the exclusion of other products that may also be suitable.

${ }^{1}$ Corresponding author. E-mail: chunxian.chen@ars.usda.gov. origins and genetic diversity of peach cultivars and elite breeding materials is needed for additional guidance in optimizing the choice of parents, to increase the efficiency of conventional breeding (Scorza et al., 1985).

Chloroplasts in flowering plants generally are inherited maternally (Birky, 1995; Hansen et al., 2007), which allows tracking of the maternal origins of plant cultivars and breeding lines (Brundu et al., 2008; Gavrilenko et al., 2013; Molina-Cano et al., 2005; Riahi et al., 2011). Many microsatellite (also known as simple sequence repeat) markers have been developed from peach nuclear genomes (Chen et al., 2014; Testolin et al., 2000; Vendramin et al., 2007; Wang et al., 2002) and are widely used in peach cultivar identification, pedigree inference, diversity analysis, and genetic mapping (Aranzana et al., 2003; Dirlewanger et al., 2007; Sitther et al., 2012; Testolin et al., 2000). Likewise, there are many reports on the development and use of chloroplast microsatellite markers in plants (Brundu et al., 2008; Gavrilenko et al., 2013; Molina-Cano et al., 2005; Phumichai et al., 2015; Riahi et al., 2011). However, apparently no chloroplast microsatellite information has been reported in peach although four generic chloroplast microsatellite loci were used to determine the parentage lineage of three full-sib european plum (Prunus domestica) cultivars (Decroocq et al., 2004). In addition, chloroplast rpL16 intron sequences were also used in a phylogeny study of 207 accessions of seventeen North American plum taxa (Shaw and Small, 2005). Recently, 11 species in Prunus and within section Prunocerasus (mostly North American plum species) were phylogenetically studied using some nuclear, mitochondrial, and chloroplast DNA markers (Chavez et al., 2016). More chloroplast microsatellite markers of known amplification 
reliability and polymorphism status are needed to facilitate maternal inheritance and phylogenetic studies on peach and other Prunus species. Several chloroplast genome sequences from Prunus species are available in the GenBank, including NC_014697 from peach (Jansen et al., 2011), NC_023956 from $P$. kansuensis, and NC_023798 from P. mume. They are valuable resources to develop in silico polymorphic microsatellites as discrepant repeat motifs among the genomes that can be validated through sequence alignment. These in silico validated microsatellite polymorphisms greatly increase the likelihood of genotyping polymorphisms among the amplicons produced in peach and other Prunus materials (Chen et al., 2014, 2015).

This study includes the following objectives: to mine and characterize microsatellites in the three Prunus chloroplast genomes to determine their in silico polymorphism status; to genotype a collection of elite peach lines and cultivars using selected microsatellites validated in silico to be polymorphic among the three chloroplasts genomes; to categorize the maternal lineages among these peach materials; and lastly, to sequence and validate all the allelic amplicons from one representative genotype in each maternal group. An evolutionary and breeding perspective in these maternal groups, along with inconsistencies in expected maternal grouping of some peach materials, is discussed.

\section{Materials and Methods}

Peach materials. A total of 736 peach accessions were genotyped, including 161 cultivars and 534 elite lines that include open-pollinated (OP) seedlings of some wild peach species. All were grafted on 'Guardian' or 'Flordaguard' rootstock. Each accession was also assigned a sample number (N001, N002, etc.) for brevity. Most accessions (713) are part of the peach breeding program collection managed at the U.S. Department of Agriculture, Agricultural Research Service (USDA-ARS) Southeastern Fruit and Tree Nut Research Laboratory in Byron, GA. Of the remaining 23 materials, 21 were acquired from the National Clonal Germplasm Repository (NCGR), Davis, CA ('Admiral Dewey', 'Belle of Georgia', 'Carmen', 'Clayton', 'Duke of York', 'Dwarf Elberta', 'Early Crawford', 'Elberta', 'George IV', 'Greensboro', 'Hiley', 'J.H. Hale', 'Late Crawford', 'Le Grand', 'Nectared 6', 'Redhaven', 'Reliance', 'Rio Oso Gem', 'Salway', 'Slappey', and 'Springtime'), whereas 'Frank' and 'Sentinel' were obtained from Bob Wells Nursery (Lindale, TX). The 736 samples contained 22 duplicates with the same names but from different trees or sources serving as controls to validate genotyping quality and compare these materials from different sources. Twelve of the duplicates are cultivars: Augustprince, Black Boy, Camden, Candy Cane (17 bud sports), Clayton, Elberta, Flameprince, GaLa, Junegold, Late Crawford, Q43183, and Redhaven. Genomic DNAs were isolated from $200 \mathrm{mg}$ young leaves using a cetyltrimethylammonium bromide protocol (Doyle and Doyle, 1987), with in-house modifications to scale down to use 2-mL Eppendorf tubes for the isolation.

IDENTIFICATION OF POLYMORPHIC MICROSATELLITES IN THREE Prunus ChlOROPlast GeNOMES. Polymorphic microsatellites were mined from three Prunus chloroplast genome sequences that were retrieved from the National Center for Biotechnology Information (NCBI). The sequences, designated as CPpe, CPka, and CPmu, respectively, were NC_014697 from $P$. persica $\mathrm{cv}$. Nemared (Jansen et al., 2011), NC_023956 from P. kansuensis (submitted to the NCBI by S.Y. Michaud, S.B. Tittes, K.G. Keepers, and N. Kane), and NC_023798 from P. mume (submitted by S. Wang, C.-W. Gao, C. Shi, and L.-Z. Gao). Microsatellite discovery and primer design were performed in the three genome sequences separately, using MISA and Primer3 with modified running parameters (Rozen and Skaletsky, 2000; Thiel et al., 2003). The microsatellite motif length and minimum repeat number pairs were changed to 1-10 2-5 3-4 4-3 5-3 6-3. The interval between any adjacent microsatellites was set to 0 , to ensure every microsatellite was found separately [i.e., to avoid any compound microsatellites (Chen et al., 2014; Thiel et al., 2003)]. The optimal primer length was set to 24 base pair (bp), and the expected microsatellite amplicon sizes were between 100 and 300 bp (Rozen and Skaletsky, 2000). Three individual tabdelimited text files were generated from CPpe, CPka, and CPmu, respectively, each containing all microsatellite motifs and primer information from the corresponding genome sequence and converted to Excel (Microsoft, Redmond, WA) files (Supplemental Table 1). An extra column was added to each of the Excel files and filled with CPpe, CPka, and CPmu, respectively, showing their chloroplast genome sources. The three files were merged into a single Excel file for comparison and identification of polymorphic microsatellites at the same loci. Polymorphic microsatellites were those with the same forward and reverse primer sequences and different motif repeat times, different amplicon lengths, or both among the three chloroplast genomes (Supplemental Table 1). Each primer was named with "CXcp," and the start position of the forward primer and the predicted amplicon size in CPpe (the two values were connected by an underscore) and also given a serial short alias starting with "CP," for brevity. For example, the values 36,648 and 290 in the primer CXcp36648_290 represent the start position of the forward primer and the predicted amplicon size in CPpe, respectively. CXcp36648_290 also had an alias, CP25. Sixteen well-distributed, in silico, polymorphic primers were selected to screen four peach accessions, three species $(P$. mume, $P$. americana, and $P$. japonica), and one plumcot $(P$. salicina $\times P$. armeniaca 'Spring Satin'), to validate the amplification reliability and polymorphism of these primers. The eight microsatellites with the most alleles detected were chosen to genotype all the materials.

Microsatellite genotyping, Allele scoring, AND MATERnAL LiNeage Grouping. Primer synthesis, polymerase chain reaction (PCR) preparation, $\mathrm{PCR}$ program, genotyping protocol, and allele scoring method were previously described Chen et al. (2014), with a change of genotyping instrument to 3500 Genetic Analyzer (Applied Biosystems, Carlsbad, CA). Owing to a M13 tail (20 bp long) added to each forward primer, the detected amplicon sizes are $\approx 20 \mathrm{bp}$ larger than the predicted product sizes, varying slightly with fluorescent dye. The microsatellite allele table and chromatograms were generated by GeneMarker (SoftGenetics, State College, PA). Maternal lineage groups of the genotyped materials were established based on the eight chloroplast alleles (sizes) detected by the eight microsatellites; materials sharing the same alleles (sizes) respectively from every of the eight microsatellites will be in the same group. The representative chosen in each group was a historically recognized cultivar (MLG-1 and MLG-2), a recently popular rootstock (MLG-3), one of the two hybrids (MLG-4), or the only material (MLG-5 to MLG-8).

Sanger Sequencing and SeQuence analysis. The PCR products of the eight primers from one representative material selected in each maternal group were purified, using the DNA 
Clean \& Concentrator kit (Zymo Research, Irvine, CA). Sanger sequencing was performed at the University of Florida, Interdisciplinary Center for Biotechnology Research, Gainesville. After extraction and verification from the chromatograph files, sequence reads from the same primer and different representatives were aligned using Clustal Omega (Sievers and Higgins, 2014) and validated manually to determine whether the length of nucleotide discrepancies [insertion/ deletion (Indel)] matched the size differences of the alleles. Single nucleotide polymorphisms were summarized, and these nucleotides were marked in each alignment.

\section{Results and Discussion}

Microsatellite polymorphisms AND LOCATIONS IN THE three Prunus Chloroplast Genomes. A total of 80,75 , and 78 microsatellites were found in the sequence of CPpe, CPka, and CPmu, respectively. Of them, 74, 68, and 73 microsatellites were successful in primer design (Table 1; Supplemental Table 1). On average, there was about one microsatellite in every two kb of the chloroplast genomes, less frequent than that in peach nuclear genome (Chen et al., 2014). A and T repeats were predominant in the three genomes, accounting for $67.8 \%$ on average (Table 1; Supplemental Fig. 1). There is one set of three adjacent microsatellites and four sets of two adjacent microsatellites sharing the same forward and reverse primers, respectively. Each set of these adjacent microsatellites was treated as one locus because the same forward and reverse primer flanks the two or three microsatellites. With the factors considered, there were 67 microsatellites with primer names in the CPpe genome, and 57 of them were polymorphic for their in silico motif, amplicon lengths, or both among the three genomes (Supplemental Table 1). These

Table 1. Number of microsatellites in the chloroplast genome of Prunus persica (CPpe), P. kansuensis (CPka), and P. mume (CPmu).

\begin{tabular}{lccc}
\hline Repeat unit size (bp) & CPpe (no.) & CPka (no.) & CPmu (no.) \\
\hline 1 & 56 & 49 & 53 \\
2 & 17 & 18 & 14 \\
3 & 0 & 0 & 1 \\
4 & 5 & 5 & 7 \\
5 & 2 & 3 & 2 \\
6 & 0 & 0 & 1 \\
Total & 80 & 75 & 78 \\
\hline
\end{tabular}

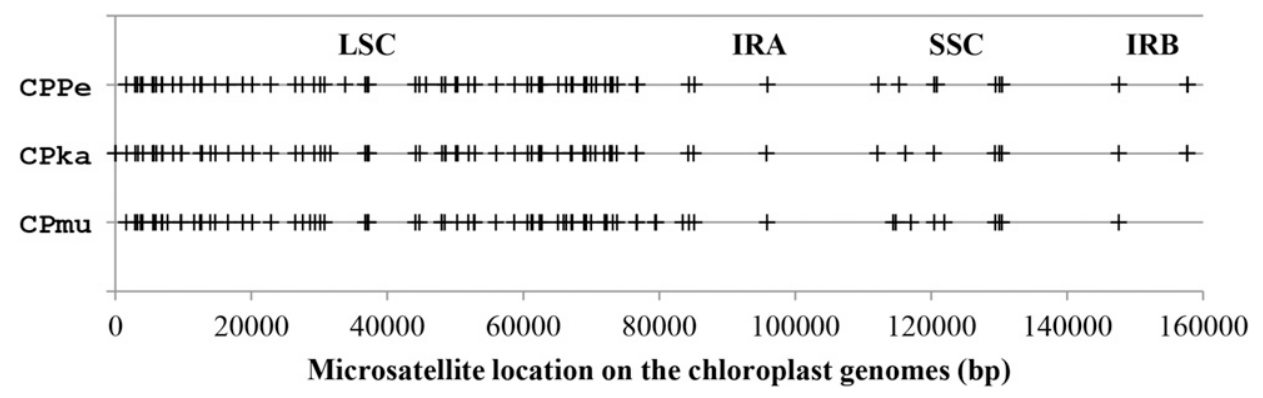

Fig. 1. Locations of the microsatellites in the chloroplast genome of Prunus persica (CPpe), P. kansuensis (CPka), and P. mume (CPmu). Each vertical bar represents a microsatellite; LSC = long single copy $(1-85,719 \mathrm{bp})$; IRA = inverted repeat A $(85,720-112,215$ bp $) ; \mathrm{SSC}=$ short single copy $(112,216-131,371 \mathrm{bp})$; IRB = inverted repeat $\mathrm{B}(131,372-157,790 \mathrm{bp})$. microsatellites with in silico polymorphism status and other mining details will be useful in any relevant genotyping and phylogenetic studies in Prunus and closely related taxa (Chavez et al., 2016; Decroocq et al., 2004; Phumichai et al., 2015; Shaw and Small, 2005).

The locations of most microsatellites are well conserved among CPpe, CPka, and CPmu (Fig. 1), as are the motifs (Supplemental Table 1). For example, 69 of the 80 CPpe microsatellites are in the long single copy (LSC) region, one in the inverted repeat A region, seven in the small single copy (SSC) region, and three in the inverted repeat B region (Fig. 1; Supplemental Fig. 2). The LSC and SSC regions play an important role in diversification of chloroplast genomes and genetic materials (Birky, 1995; Gavrilenko et al., 2013; Hansen et al., 2007; Molina-Cano et al., 2005). In another categorization, 49 of the 80 microsatellites are in noncoding sequences, 15 in exons, and 16 in introns (Supplemental Table 1).

Maternal lineages of Peach Materials BaSed ON THE EIGHT MOST POLYMORPHIC MICROSATELLITES. Screening of 16 in silico polymorphic microsatellite primers generated three to seven alleles (amplicons of different sizes) (Table 2). Based on the numbers of alleles detected, the eight most polymorphic microsatellites were used to genotype and subsequently to classify the 736 materials into eight unique MLGs (Tables 3 and 4; Supplemental Table 2; Supplemental Fig. 3).

MLG-1 contained the majority of peach cultivars (111) and selections (354) genotyped (Table 4; Supplemental Table 2). 'Chinese Cling' is used as the representative because of its status as the progenitor clone for most of the cultivars in the group. The predominance is expected based on the heavy use of 'Chinese Cling' offspring such as 'Elberta' in early breeding work (Myers et al., 1989; Scorza et al., 1985). Almost all the cultivars in this group, for which pedigrees are available, descended from 'Elberta', 'J.H. Hale' (thought to be an 'Elberta' seedling), or 'Late Crawford' (an old American peach presumably of European ancestry, see below) via 'Rio Oso Gem' (Okie, 1998). Only two cultivars and several selections in this group were inconsistent with their pedigree-inferred maternal groups. 'Majestic' and several selections descended from it, supposedly traced back to 'St. John' (see MLG-2), an old American cultivar dating back to the 1860s. 'Red Bird Cling' was thought to be a seedling of 'Heath Cling' (MLG-2). One selection tracing back to 'Admiral Dewey' (MLG-2) via 'LaPremiere' and 'Redglobe' and another to 'Sunprince' (MLG-2) appeared in this group. Three other selections supposedly belonged to MLG-1 but fell in MLG-3.

MLG-2 consists of 41 cultivars and 152 selections (Table 4; Supplemental Table 2). The group includes 'Heath Cling' (used as the representative for this group because it is one of the oldest cultivars in the group) and most other old (i.e., pre-'Elberta') American cultivars and their European progenitors, which presumably trace back to peaches introduced into Europe over the last two millennia (Faust and Timon, 1995). Most cultivars with known pedigrees were descended from 'Loring', thought to be a seedling of 'Frank' [itself 


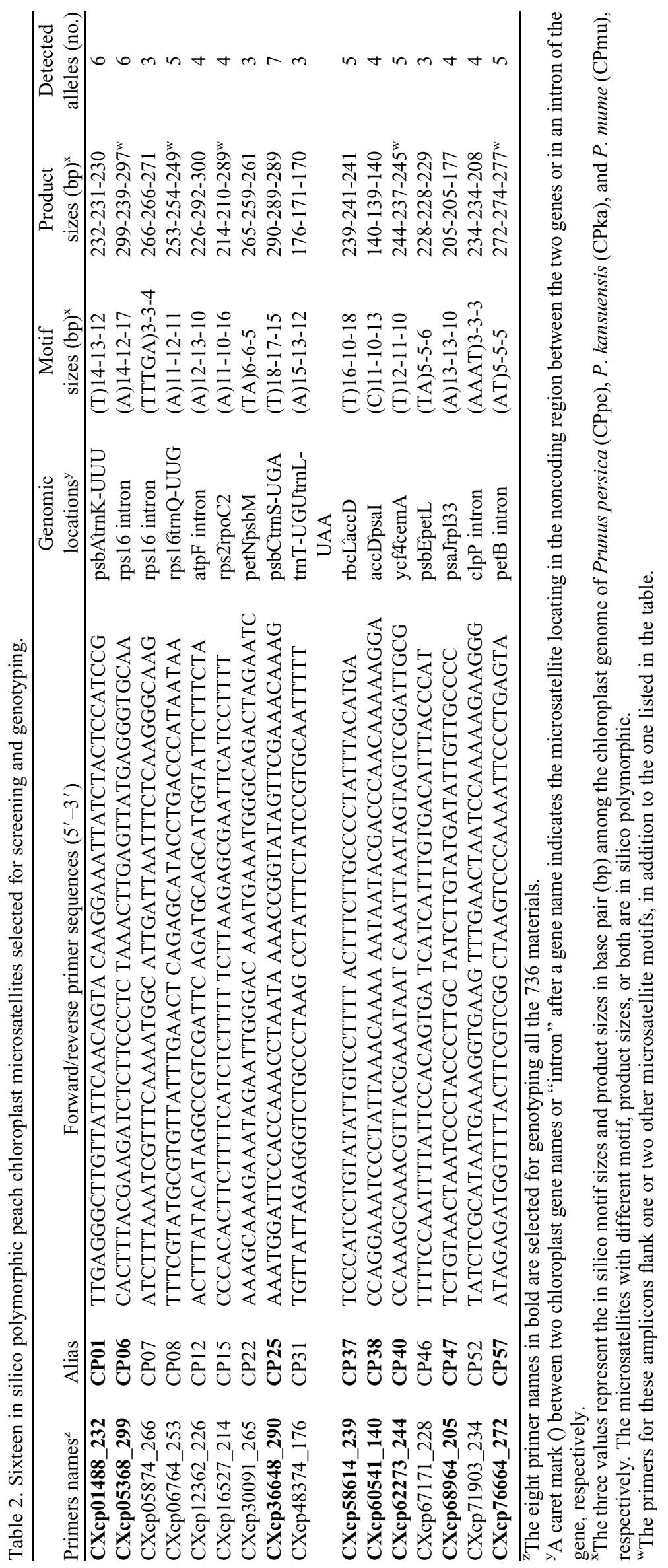


a supposed seedling of 'Elberta' (see MLG-1)] (Okie, 1998). 'Sunprince' and its selections which trace back to 'Redglobe' and 'Admiral Dewey' landed in this group as would be expected based on the old origin of 'Dewey'. Several cultivars in this group are ornamentals, including 'MacDonald', 'Helen Borchers', and 'White English', or have ornamental maternal ancestors such as 'Flordaguard' (via PI65821) and 'Candy Cane' (via 'Shidaremomo' from Japan). Several were selections from 'Naturals', feral seedlings found growing wild, which could trace back to pre-'Chinese Cling' peaches. Inconsistencies exist in one cultivar and a few selections in this group. 'Saturn', a flat peach, is reportedly descended from 'J.H. Hale' (MLG-1). Three selections with pedigrees tracing back to 'J.H. Hale' also

Table 3. The sizes of alleles detected in eight representative peach materials for eight different maternal lineage groups (MLG) by eight chloroplast (CP) primers.

\begin{tabular}{|c|c|c|c|c|c|c|c|c|c|}
\hline \multirow[b]{3}{*}{ MLG } & \multirow[b]{3}{*}{ Representative } & \multicolumn{8}{|c|}{ Primers $^{z}$} \\
\hline & & $\overline{\mathrm{CP} 01}$ & CP06 & CP25 & CP37 & CP38 & CP40 & CP47 & CP57 \\
\hline & & \multicolumn{8}{|c|}{ (base pair) } \\
\hline MLG-1 & Chinese Cling & 250 & 313 & 308 & 263 & 161 & 262 & 225 & 296 \\
\hline MLG-2 & Heath Cling & 255 & 316 & 310 & 264 & 158 & 264 & 225 & 295 \\
\hline MLG-3 & Guardian & 254 & 314 & 309 & 264 & 160 & 263 & 224 & 295 \\
\hline MLG-4 & Flordaking & 254 & 315 & 309 & 265 & 158 & 264 & 225 & 295 \\
\hline MLG-5 & Reliance & 255 & 310 & 310 & 264 & 158 & 264 & 225 & 295 \\
\hline MLG-6 & Prunus tangutica ${ }^{\mathrm{y}}$ & 253 & 310 & 301 & 254 & 142 & 267 & 220 & 298 \\
\hline MLG-7 & Prunus davidiana ${ }^{\mathrm{x}}$ & 255 & 317 & 303 & 262 & 160 & 260 & 222 & 295 \\
\hline MLG-8 & Prunus mira & 261 & 317 & 302 & 262 & 162 & 260 & 224 & 294 \\
\hline
\end{tabular}

${ }^{\mathrm{z}}$ The primer names for $\mathrm{CP} 01, \mathrm{CP} 06, \mathrm{CP} 25, \mathrm{CP} 37, \mathrm{CP} 38, \mathrm{CP} 40, \mathrm{CP} 47$, and CP57 are CXcp01488_232, CXcp05368_299, CXcp36648_290, CXcp58614_239, CXcp60541_140, CXcp62273_244, CXcp68964_205, and CXcp76664_272, respectively.

${ }^{\mathrm{y}} \mathrm{A}$ hybrid of this species.

${ }^{\mathrm{x}}$ An open-pollinated seedling of this species. fell into this group, as did two selections tracing 'Late Crawford' via 'Rio Oso Gem' (MLG-1).

MLG-3 consists of only 30 accessions, mostly 'Guardian' and its seedlings (rootstock descended from S-37, an ornamental/rootstock selection from Stribling's Nursery, CA) or other 'S-37' descendants (Okie et al., 1994), 'Kikomo' OP (Q36019e, ornamental from Japan) and 'Hong Chui Zhi' (Q43183, weeping ornamental from China); this group likely is the maternal source of some ornamental peaches. One selection tracing back to 'Flameprince' (MLG-1) also was in this group.

MLG-4 contains only 'Flordaking' and MLG-5 only 'Reliance', representing their unique maternal sources. 'Flordaking' descended from 'Okinawa', a low-chill clone of unknown origin that was obtained from the island after which it was named (Andrews et al., 1979; Okie, 1998). 'Reliance' is a hybrid of 'Minnesota PH-04559' $\times$ 'Meredith' released in the New Hampshire Agriculture Experiment Station, which is extremely bud hardy and able to fruit in cold areas (Okie, 1998). PH-04559 probably descended from peaches growing at the northern limit of peach production, so it would be interesting to find the parentage of this special selection.

The next three groups, MLG-6 to MLG-8, consist of materials from three Prunus species. MLG-6 consists of two selections derived from $P$. tangutica, a species with dryflesh, flattened fruit usually classified with almond ( $P$. dulcis),

Table 4. Peach cultivars or lines belonging to each maternal lineage group (MLG). Cultivars tested in duplicate are underlined.

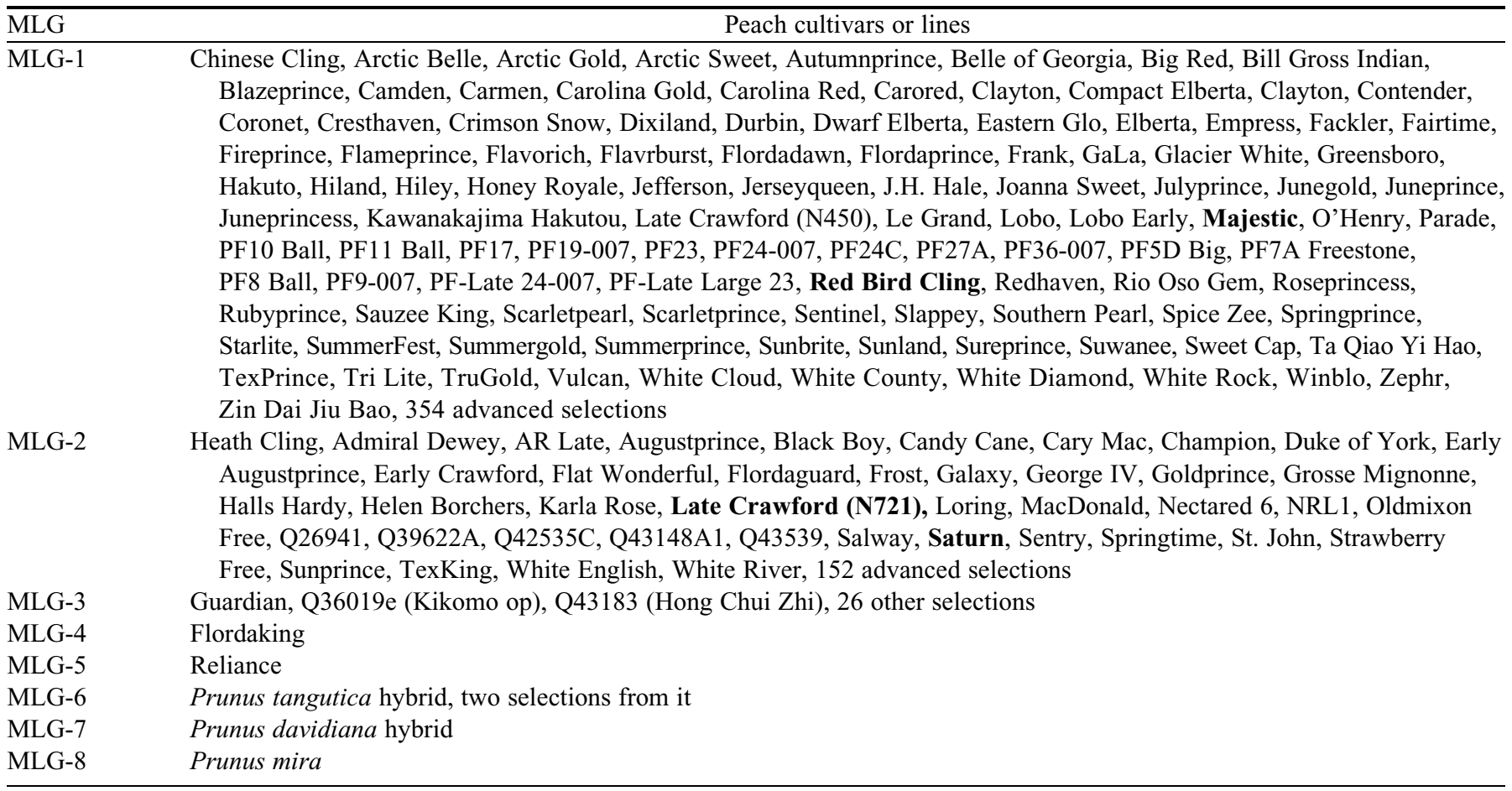

Cultivars in bold appear inconsistent with their recorded pedigree $(\mathrm{OP}=$ open-pollinated). 
and found in Sichuan, China. It is commonly named 'Tang Gu Te Bian Tao' or 'Xi Kang Bian Tao'. MLG-7 consists of an OP seedling of $P$. davidiana (a wild Chinese peach species) which appears to be a hybrid with peach. MLG- 8 is represented by a clone of $P$. mira, a peach species native to China and Nepal.

Among the duplicated samples, all the sets, including 'Augustprince', 'Black Boy', 'Camden', 'Candy Cane', 'Clayton', 'GaLa', 'Elberta', 'Flameprince', and 'Redhaven', yielded identical genotyping data among the eight primers, except for the two 'Late Crawford' samples. 'Late Crawford' is a very old freestone cultivar named in New Jersey in the early 1800 s (Okie, 1998). 'Late Crawford' at Byron belongs to MLG-1, the 'Chinese Cling' group, whereas the clone from the NCGR belongs to MLG-2, the European peach group. One would expect 'Late Crawford' to fall into MLG-2 based on its origin, but it is odd that 'Rio Oso Gem' (a 1931 seedling thought to be from 'Late Crawford') and other cultivars that descended it, all fell into the MLG-1. In the case of historical cultivars such as Late Crawford, it is difficult to tell if a current clone is identical to that which existed over a century ago, although the clone at Byron matches the historical description based on flower and fruit characters. Sometimes seedlings of a cultivar retained the parental name. In other cases, parentage was presumed or inferred from seedling performance. Cultivars grown at Byron appeared to fit the published descriptions, accounting for differences in climate, for 'LaPremiere' (MLG-1), 'Majestic' (MLG-1), 'Red Bird Cling' (MLG-1), and 'Saturn' (MLG-2), although based on their pedigree they were expected to group in MLG-1, MLG-2, MLG-2, and MLG-1, respectively. There are several other possible reasons for the inconsistent cases. Pedigree information, especially on old cultivars, may be anecdotal and inaccurate. Cross records for pedigrees can contain transcriptional errors, and trees used for crossing and propagation can be mislabeled. Bud failure results in rootstock growing as scion. For example, three selections expected to be in MLG-1 but falling in MLG-3 were proved to be 'Guardian' rootstock seedlings (data not shown). Genotyping of these inconsistent materials using nuclear microsatellite markers shall make it possible to infer their parentage, as reported in other studies (Aranzana et al., 2003; Chen and Okie, 2015; Decroocq et al., 2004; Sitther et al., 2012; Testolin et al., 2000).

DisCREPANT NUCLEOTIDES IN MICROSATELLITE AMPLICONS AMONG THE MATERNAL LINEAGES. Discrepant nucleotides were revealed by sequencing and alignment of the microsatellite

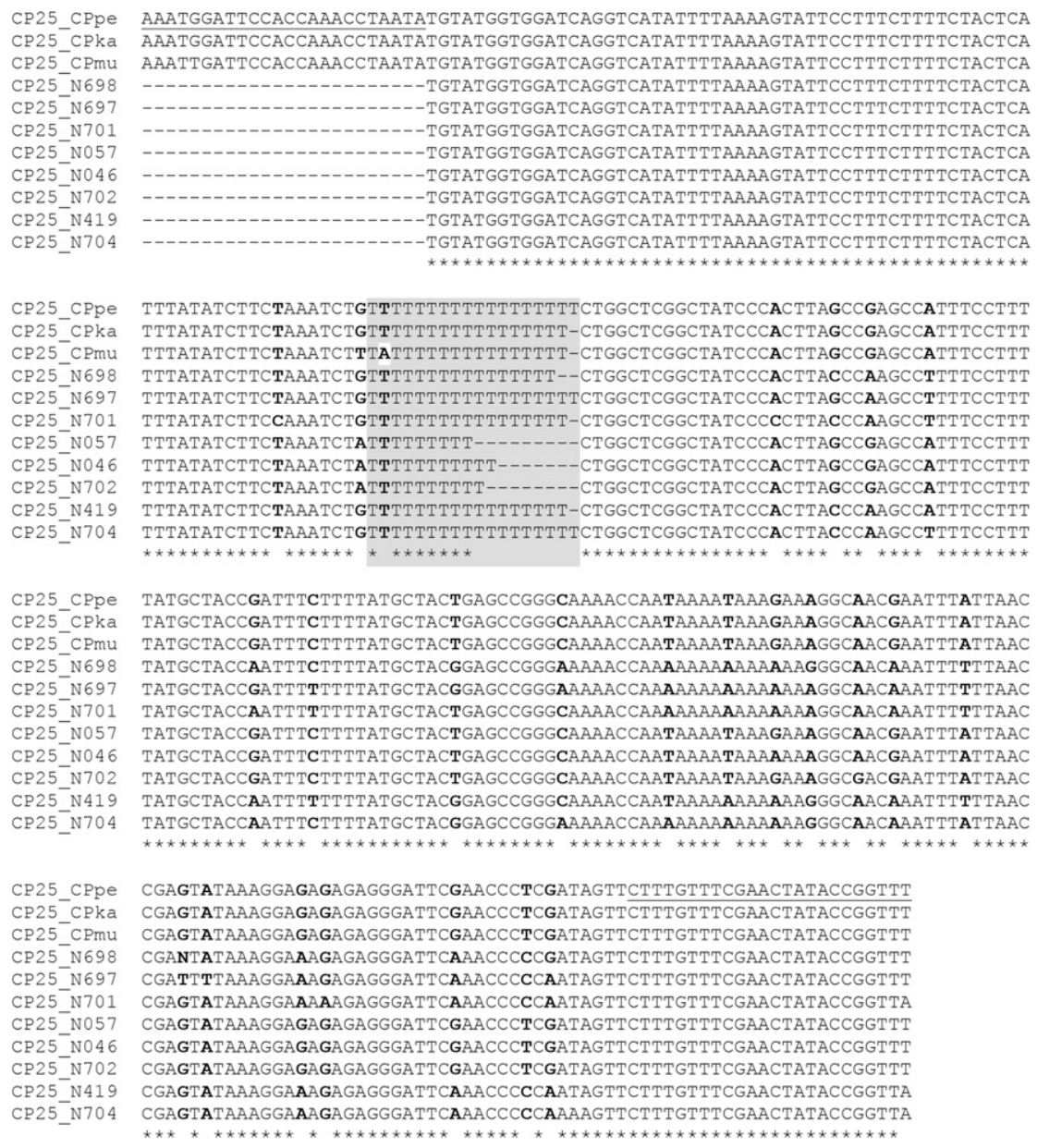

Fig. 2. Alignment of amplicon sequences with primer CXcp36648_290 (CP25) in ‘Chinese Cling' (N698), 'Heath Cling' (N697), 'Guardian’ (N701), 'Flordaking' (N419), 'Reliance' (N704), Prunus tangutica hybrid (N057), P. davidiana open-pollinated (N046), and P. mira (N702) peaches. The sequences corresponding to the amplicons in CPpe (P. persica), CPka (P. kansuensis), and CPmu (P. mume) genomes, named as CP25_CPper, CP25_CPka, and CP25_CPmu, were included in the alignment. The polymorphic microsatellite nucleotides were grayed, and the forward and reverse primer sequences were underlined. CP25_CPmu has the same amplicon size of 309 detected in genotyping, but only $\mathrm{T}_{15}$ is mined because of an A (ungrayed) substitution in the microsatellite. In the asterisk line, asterisks represent consensus nucleotides, and spaces represent discrepant (polymorphic) nucleotides. 
amplicons among the eight peach materials representing the eight MLGs (Fig. 2). The length differences among the microsatellite repeats matched well with those among the sizes of these alleles detected by the microsatellite genotyping (Table 3; Supplemental Fig. 3). Taking the primer CXcp36648_290 (CP25) as an example, 'Heath Cling' (CP25_N697) and 'Reliance' (CP25_N704), together with 'Nemared' (CP25_CPpe), have the $\mathrm{T}$ nucleotide repeating 18 times $\left(\mathrm{T}_{18}\right)$ at the microsatellite locus (Fig. 2) and yielding the largest allele size of 310 bp detected in genotyping (Table 2). The next are 'Guardian' (CP25_N701), 'Flordaking' (CP25_N419), and CP25_CPka, which have the repeat of $\mathrm{T}_{17}$ and the amplicon size of 309 . CP25_CPmu has the same amplicon size of 309 , but only $\mathrm{T}_{15}$ is mined because of one A substitution in the microsatellite. Likewise, 'Chinese Cling' (CP25_N698) has $\mathrm{T}_{16}$ and 308; $P$. davidiana OP (CP25_N046) has $\mathrm{T}_{11}$ and 303; P. mira (CP25_N702) has $\mathrm{T}_{10}$ and 302 ; and $P$. tangutica hybrid (CP25_N057) has $\mathrm{T}_{9}$ and 301. The $\mathrm{T}$ deletions/insertions at this satellite locus yield the length polymorphisms so do those at the other microsatellites. In addition, various numbers of SNPs were also found within the sequences of amplicons amplified by the eight primers (Fig. 2; Supplemental Table 3).

Cultivated peaches in the United States show low genetic diversity (Aranzana et al., 2003; Chen et al., 2014; Scorza et al., 1985; Sitther et al., 2012). Likewise, they may also have limited maternal origins, as this study has demonstrated. Peach is selfcompatible, and conventional hybridization remains the main approach to breeding peach cultivars. The in silico chloroplast microsatellite polymorphisms and genotyping results in these peach materials provide insights into chloroplast genome variations and can guide combinations of parents with known maternal origins. In addition, the amplicon length polymorphisms only distinguished eight maternal groups; however, SNPs are common in these small amplicons. Further exploitation of these nucleotide polymorphisms will lead to more maternal subgroups and supply sufficient markers for a whole genome association study for chloroplast-controlled traits.

\section{Literature Cited}

Andrews, C.P., W.B. Sherman, and P.M. Lyrene. 1979. Flordaking peach. HortScience 14:81-82.

Aranzana, M.J., J. Carbo, and P. Arus. 2003. Microsatellite variability in peach [Prunus persica (L.) Batsch]: Cultivar identification, marker mutation, pedigree inferences and population structure. Theor. Appl. Genet. 106:1341-1352.

Birky, C.W. 1995. Uniparental inheritance of mitochondrial and chloroplast genes - mechanisms and evolution. Proc. Natl. Acad. Sci. USA 92:11331-11338.

Brundu, G., R. Lupi, I. Zapelli, T. Fossati, G. Patrignani, I. Camarda, F. Sala, and S. Castiglione. 2008. The origin of clonal diversity and structure of Populus alba in Sardinia: Evidence from nuclear and plastid microsatellite markers. Ann. Bot. (Lond.) 102:997-1006.

Cantin, C.M., Y. Gogorcena, and M.A. Moreno. 2010. Phenotypic diversity and relationships of fruit quality traits in peach and nectarine [Prunus persica (L.) Batsch] breeding progenies. Euphytica 171:211-226.

Chavez, D.J., T.G. Beckman, and J.X. Chaparro. 2016. Identifying the North American plum species phylogenetic signal using nuclear, mitochondrial, and chloroplast DNA markers. J. Amer. Soc. Hort. Sci. 141:623-644.

Chen, C., C.H. Bock, T.G. Beckman, B.W. Wood, and W.R. Okie. 2015. Genomic and genotyping characterization of haplotypebased polymorphic microsatellites in Prunus. J. Genet. Genome Res. 2:014.
Chen, C., C.H. Bock, W.R. Okie, F.G. Gmitter, S. Jung, D. Main, T.G. Beckman, and B.W. Wood. 2014. Genome-wide characterization and selection of expressed sequence tag simple sequence repeat primers for optimized marker distribution and reliability in peach. Tree Genet. Genomes 10:1271-1279.

Chen, C. and W.R. Okie. 2015. Novel peach flower types in a segregating population from 'Helen Borchers'. J. Amer. Soc. Hort. Sci. 140:172-177.

Chen, C.X., J.H. Bai, W.R. Okie, and A. Plotto. 2016. Comparison of fruit characters and volatile components in peach-to-nectarine mutants. Euphytica 209:409-418.

Decroocq, V., L.S. Hagen, M.G. Fave, J.P. Eyquard, and A. Pierronnet. 2004. Microsatellite markers in the hexaploid Prunus domestica species and parentage lineage of three European plum cultivars using nuclear and chloroplast simple-sequence repeats. Mol. Breed. 13:135-142.

Dirlewanger, E., P. Cosson, K. Boudehri, C. Renaud, G. Capdeville, Y. Tauzin, F. Laigret, and A. Moing. 2007. Development of a secondgeneration genetic linkage map for peach [Prunus persica (L.) Batsch] and characterization of morphological traits affecting flower and fruit. Tree Genet. Genomes 3:1-13.

Doyle, J.J. and J.L. Doyle. 1987. A rapid DNA isolation procedure for small quantities of fresh leaf tissue. Phytochem. Bull. 19:11-15.

Faust, M. and B. Timon. 1995. Origin and dissemination of peach. Hort. Rev. 17:331-379.

Gavrilenko, T., O. Antonova, A. Shuvalova, E. Krylova, N. Alpatyeva, D.M. Spooner, and L. Novikova. 2013. Genetic diversity and origin of cultivated potatoes based on plastid microsatellite polymorphism. Genet. Resources Crop Evol. 60:1997-2015.

Hansen, A.K., L.K. Escobar, L.E. Gilbert, and R.K. Jansen. 2007. Paternal, maternal, and biparental inheritance of the chloroplast genome in Passiflora (Passifloraceae): Implications for phylogenetic studies. Amer. J. Bot. 94:42-46.

Jansen, R.K., C. Saski, S.B. Lee, A.K. Hansen, and H. Daniell. 2011. Complete plastid genome sequences of three rosids (Castanea, Prunus, Theobroma): Evidence for at least two independent transfers of rpl22 to the nucleus. Mol. Biol. Evol. 28:835-847.

Lohse, M., O. Drechsel, S. Kahlau, and R. Bock. 2013. OrganellarGenomeDRAW - A suite of tools for generating physical maps of plastid and mitochondrial genomes and visualizing expression data sets. Nucleic Acids Res. 41:W575-W581.

Molina-Cano, J.L., J.R. Russell, M.A. Moralejo, J.L. Escacena, G. Arias, and W. Powell. 2005. Chloroplast DNA microsatellite analysis supports a polyphyletic origin for barley. Theor. Appl. Genet. 110: 613-619.

Myers, S.C., W.R. Okie, and G. Lightner. 1989. The Elberta peach. Fruit Var. J. 43:130-138.

Okie, W.R. 1998. Handbook of peach and nectarine varieties: Performance in the southeastern United States and index of names. U.S. Dept. Agr., Agr. Hdbk. No. 714.

Okie, W.R., T.G. Beckman, A.P. Nyczepir, G.L. Reighard, W.C. Newall, and E.I. Zehr. 1994. BY520-9, a peach rootstock for the southeastern United States that increases scion longevity. HortScience 29:705-706.

Phumichai, C., T. Phumichai, and A. Wongkaew. 2015. Novel chloroplast microsatellite (cpssr) markers for genetic diversity assessment of cultivated and wild hevea rubber. Plant Mol. Biol. Rpt. 33:1486-1498.

Riahi, L., N. Zoghlami, V. Laucou, A. Mliki, and P. This. 2011. Use of chloroplast microsatellite markers as a tool to elucidate polymorphism, classification and origin of Tunisian grapevines. Sci. Hort. 130:781-786.

Rozen, S. and H. Skaletsky. 2000. Primer3 on the WWW for general users and for biologist programmers. Methods Mol. Biol. 132:365-386.

Scorza, R., S.A. Mehlenbacher, and G.W. Lightner. 1985. Inbreeding and coancestry of freestone peach cultivars of the eastern United States and implications for peach germplasm improvement. J. Amer. Soc. Hort. Sci. 110:547-552. 
Shaw, J. and R.L. Small. 2005. Chloroplast DNA phylogeny and phylogeography of the North American plums (Prunus subgenus Prunus section Prunocerasus, Rosaceae). Amer. J. Bot. 92:20112030.

Sievers, F. and D.G. Higgins. 2014. Clustal Omega, accurate alignment of very large numbers of sequences. Methods Mol. Biol. 1079:105-116.

Sitther, V., D.P. Zhang, S.A. Dhekney, D.L. Harris, A.K. Yadav, and W.R. Okie. 2012. Cultivar identification, pedigree verification, and diversity analysis among peach cultivars based on simple sequence repeat markers. J. Amer. Soc. Hort. Sci. 137:114-121.

Testolin, R., T. Marrazzo, G. Cipriani, R. Quarta, I. Verde, M.T. Dettori, M. Pancaldi, and S. Sansavini. 2000. Microsatellite DNA in peach
(Prunus persica L. Batsch) and its use in fingerprinting and testing the genetic origin of cultivars. Genome 43:512-520.

Thiel, T., W. Michalek, R.K. Varshney, and A. Graner. 2003. Exploiting EST databases for the development and characterization of gene-derived SSR-markers in barley (Hordeum vulgare L.). Theor. Appl. Genet. 106:411-422.

Vendramin, E., M.T. Dettori, J. Giovinazzi, S. Micali, R. Quarta, and I. Verde. 2007. A set of EST-SSRs isolated from peach fruit transcriptome and their transportability across Prunus species. Mol. Ecol. Notes 7:307-310.

Wang, Y., L.L. Georgi, T.N. Zhebentyayeva, G.L. Reighard, R. Scorza, and A.G. Abbott. 2002. High-throughput targeted SSR marker development in peach (Prunus persica). Genome 45:319-328. 


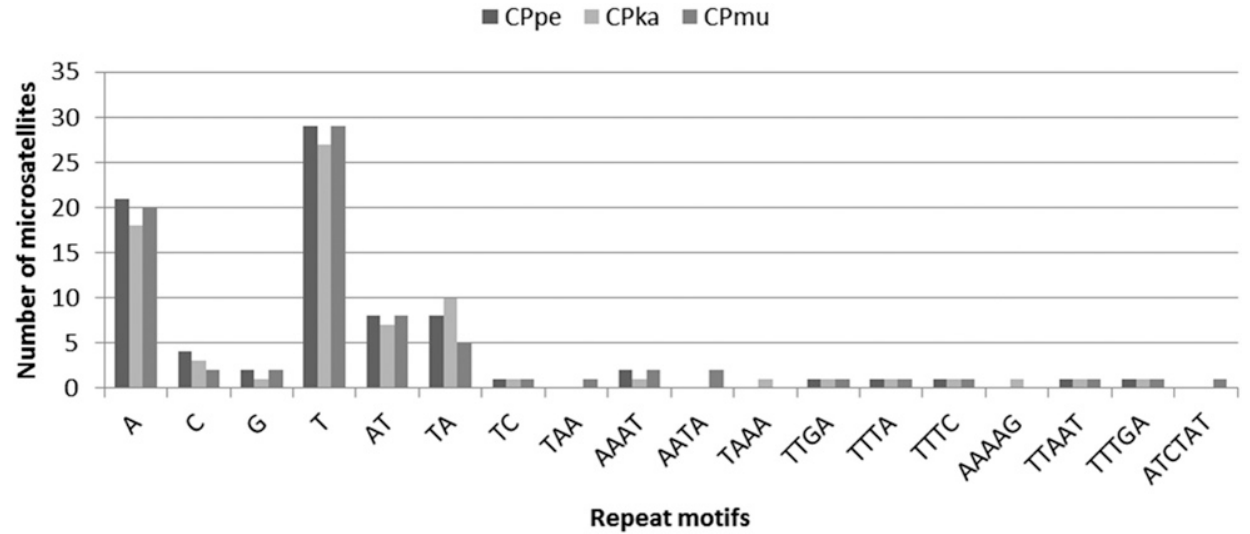

Supplemental Fig. 1. Number of microsatellites with different repeat motifs found in the chloroplast genome of Prunus persica (CPpe), P. kansuensis (CPka), and P. mume (CPmu), respectively. 


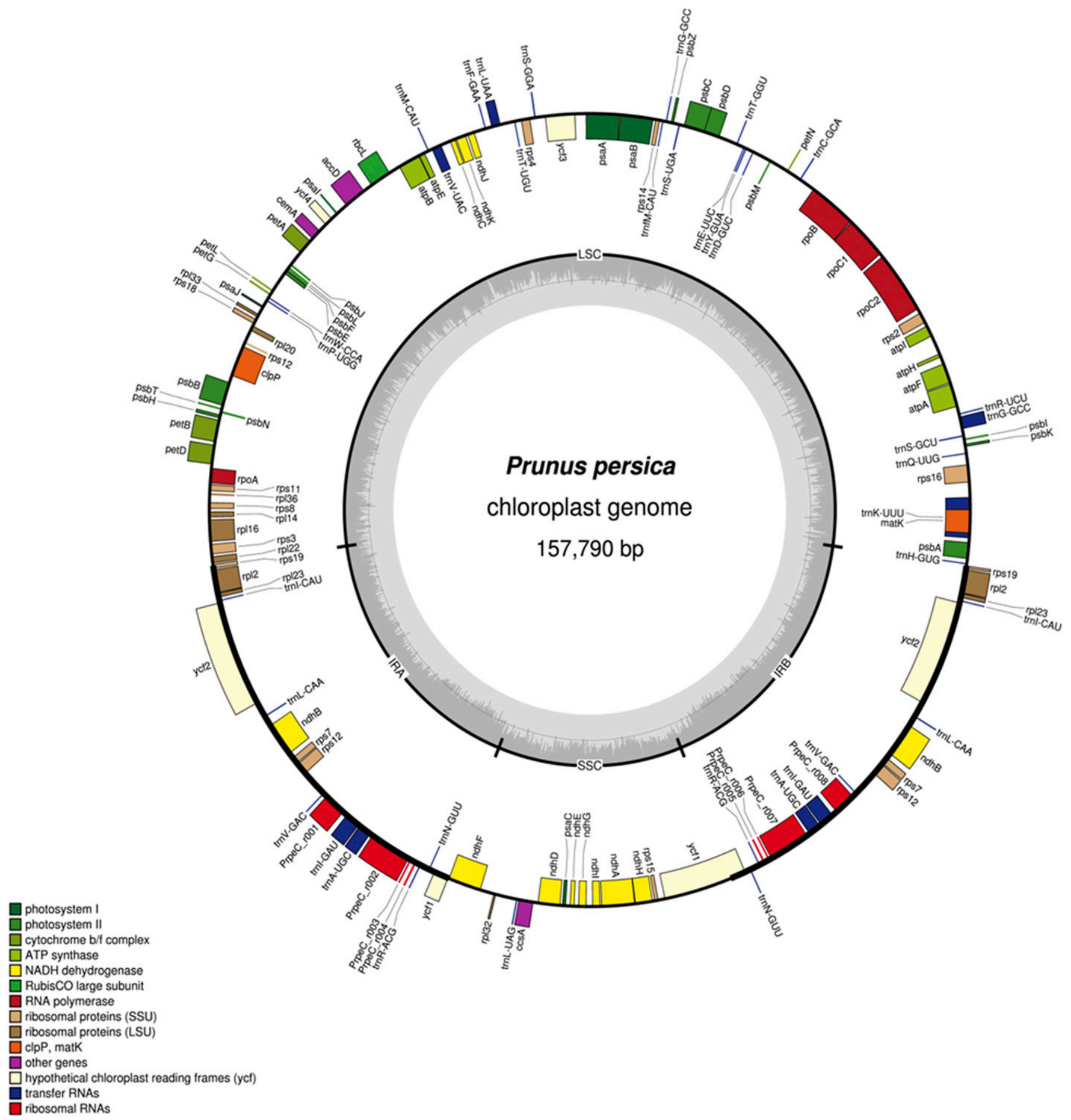

Supplemental Fig. 2. Peach chloroplast genome physical map generated by OrganellarGenomeDRAW (Lohse et al., 2013). All genes and the regions of long single copy (LSC), inverted repeat A (IRA), small single copy (SSC), and inverted repeat B (IRB) were proportionally displayed. 


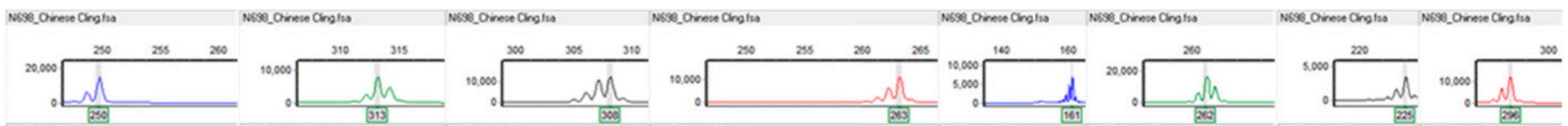

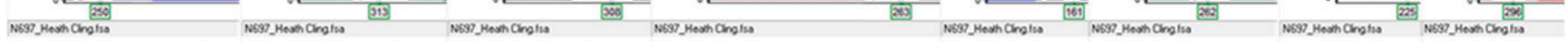
20.000

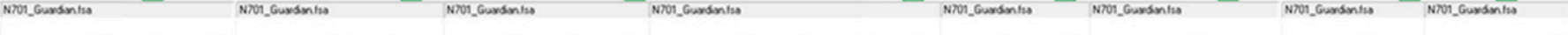

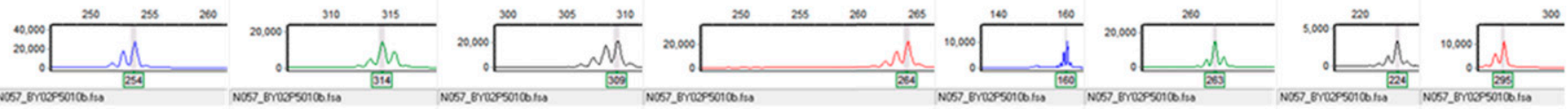

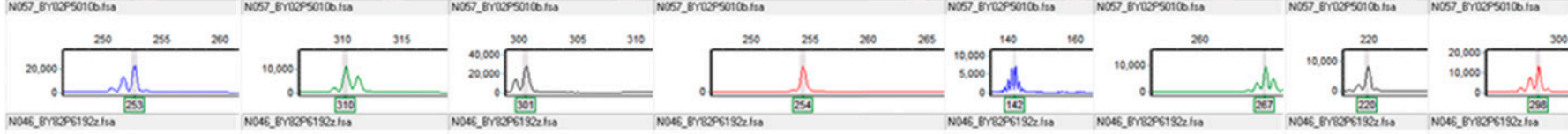

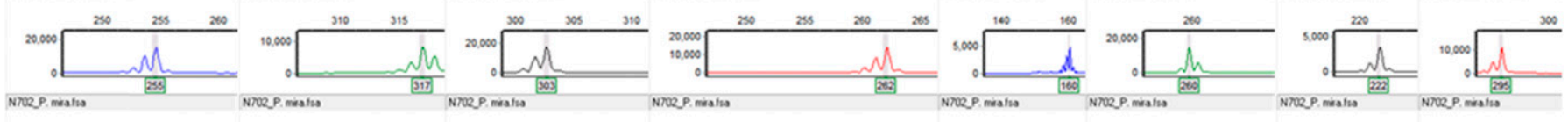

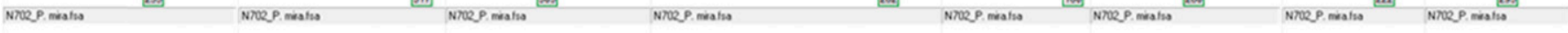

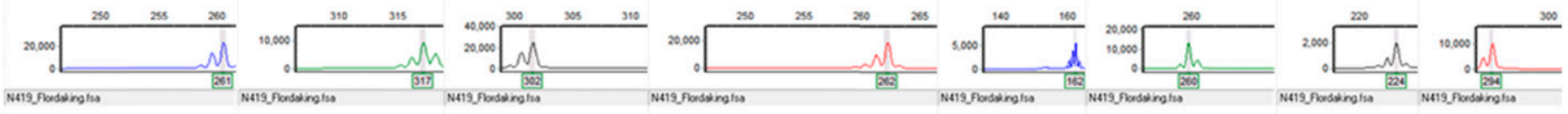

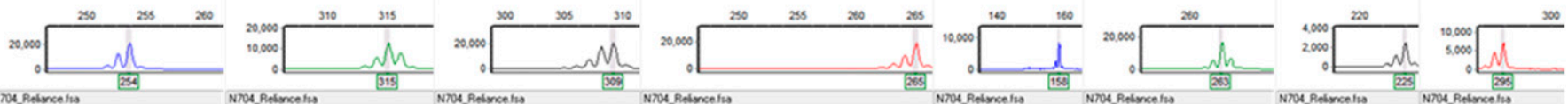

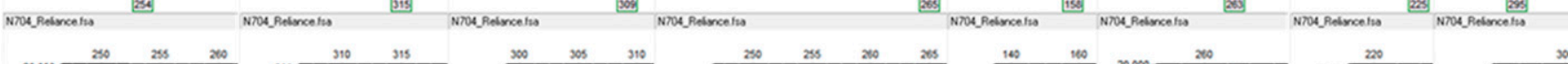

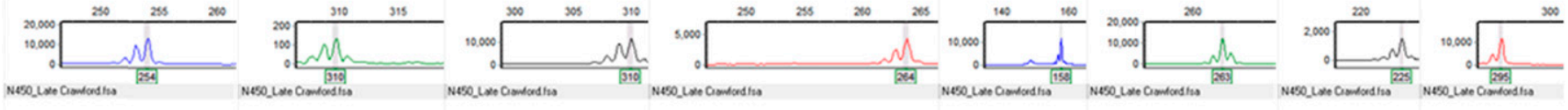

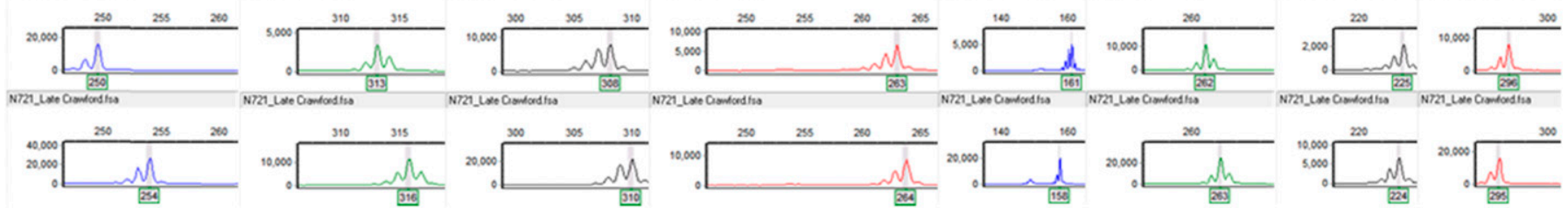

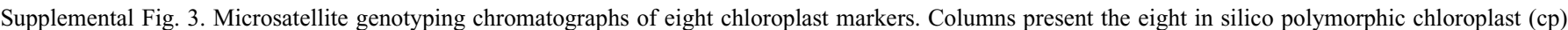
microsatellite markers (alias in the parentheses): CXcp01488_232 (CP01), CXcp05368_299 (CP06), CXcp36648_290 (CP25), CXcp58614_239 (CP37), CXcp60541_140(CP38), CXcp62273_244 (CP40), CXcp68964_205 (CP47), and CXcp76664_272 (CP57). Rows present the eight peach materials representing the eight maternal lineage groups [MLGs (alias names in the parentheses)]: 'Chinese Cling' (N698), 'Heath Cling' (N697), 'Guardian' (N701), $P$. tangutica hybrid (N057), P. davidiana open-pollinated (N046), P. mira (N702), 'Flordaking' (N419), and 'Reliance' (N704), and two conflicting 'Late Crawford' (N450 and N721). These peaks are the chloroplast alleles among the materials detected by the microsatellite markers. The allele sizes are marked under the baseline of the peaks and displayed in Table 3. 


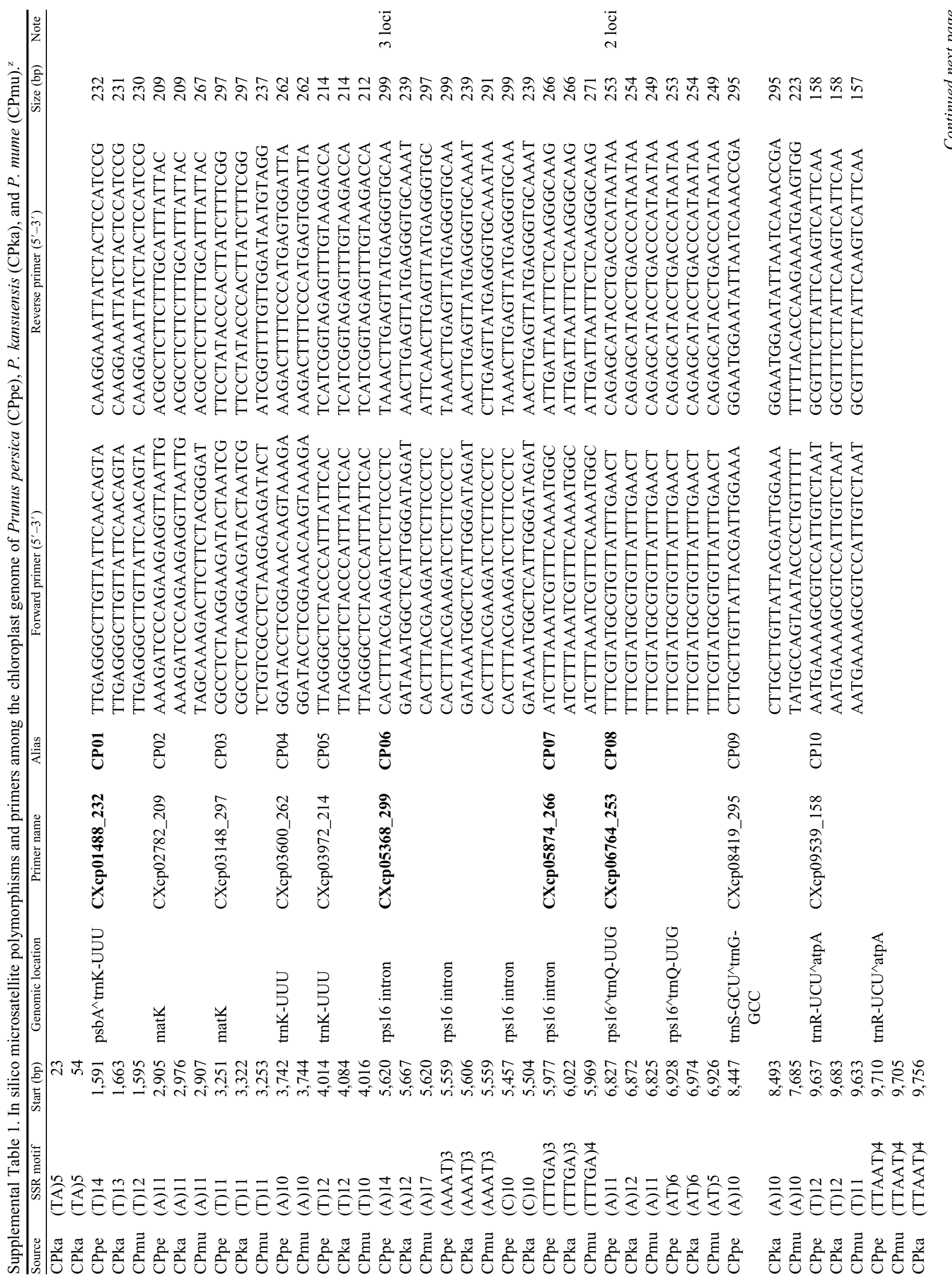




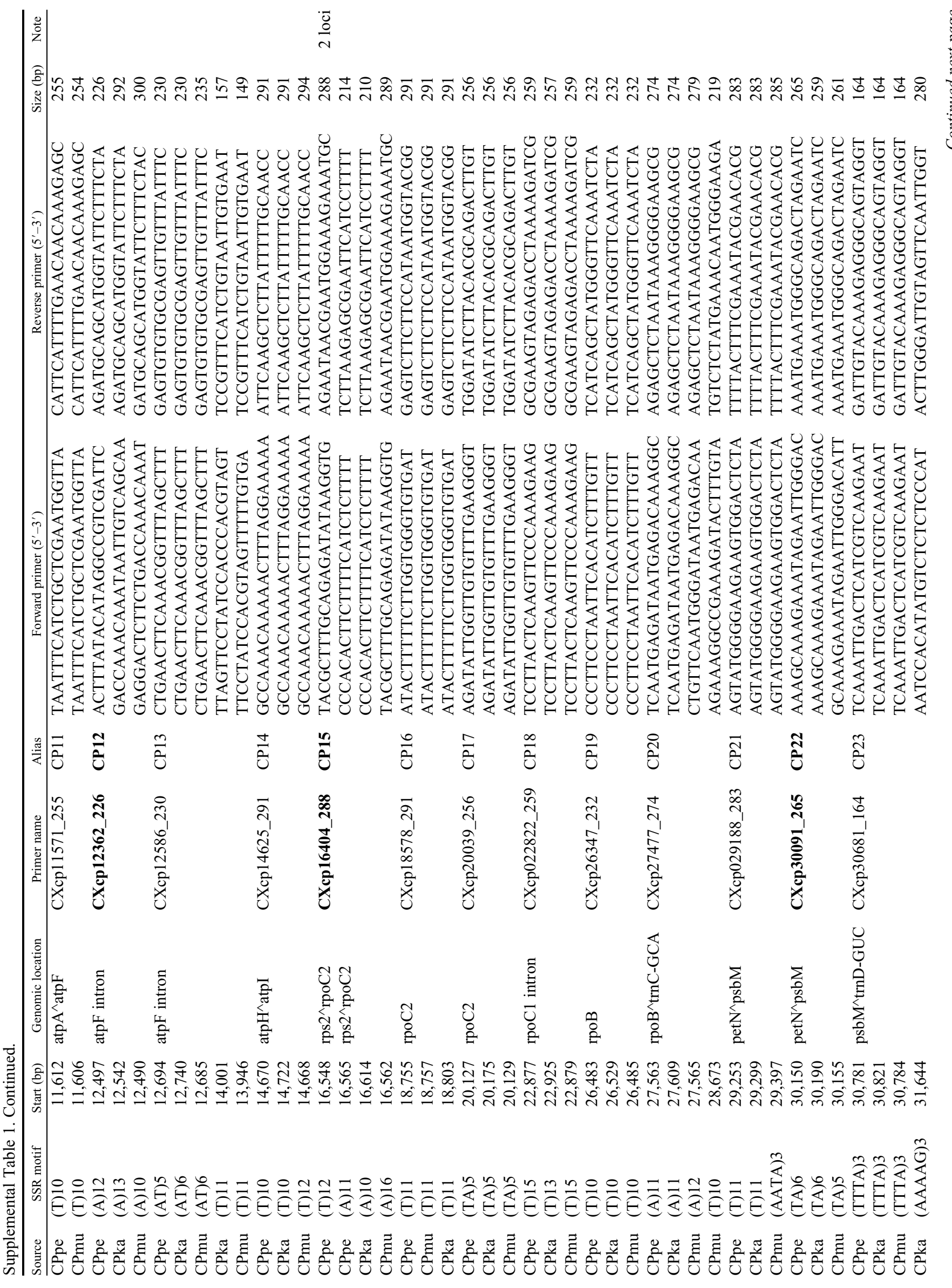




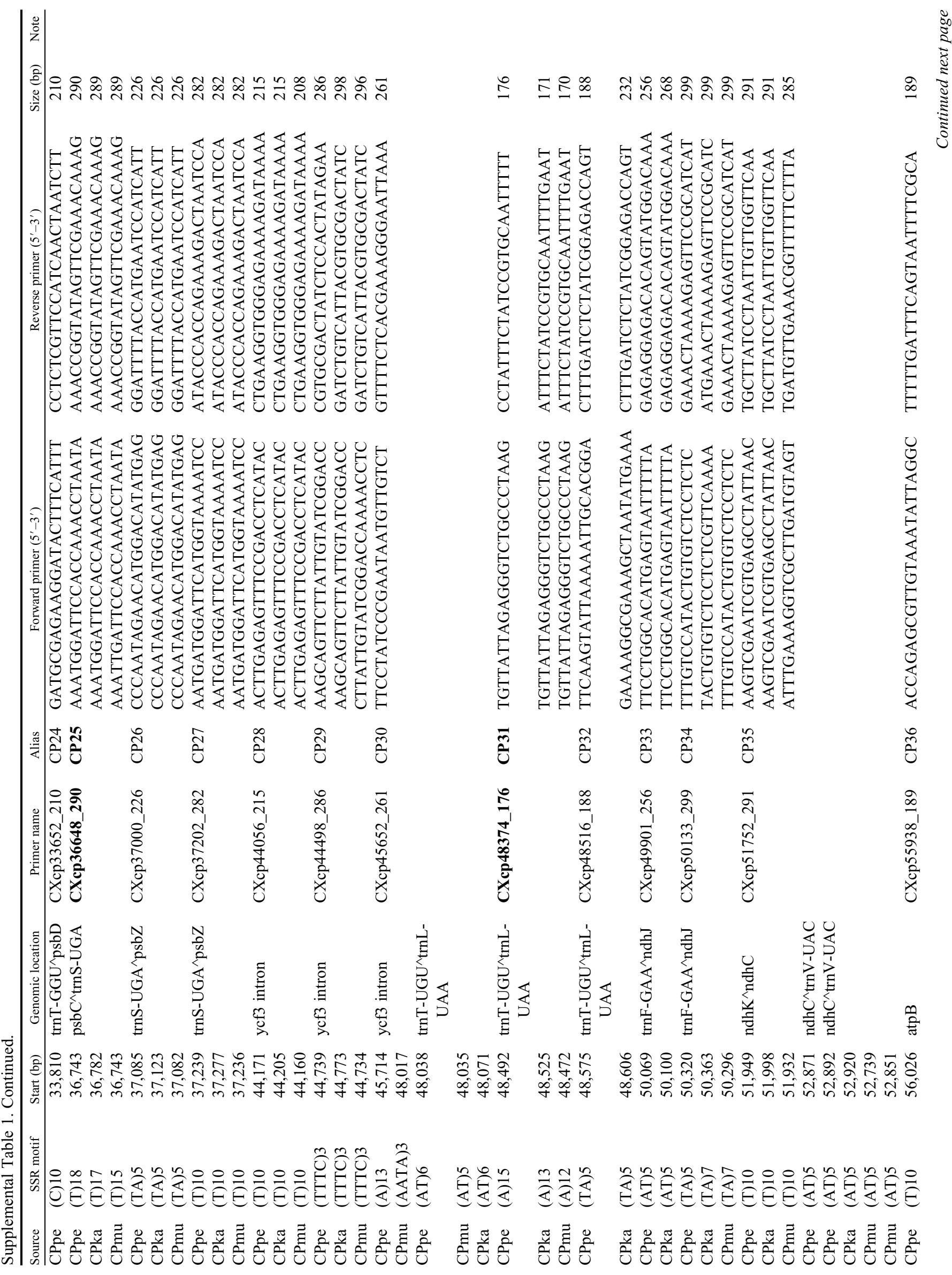




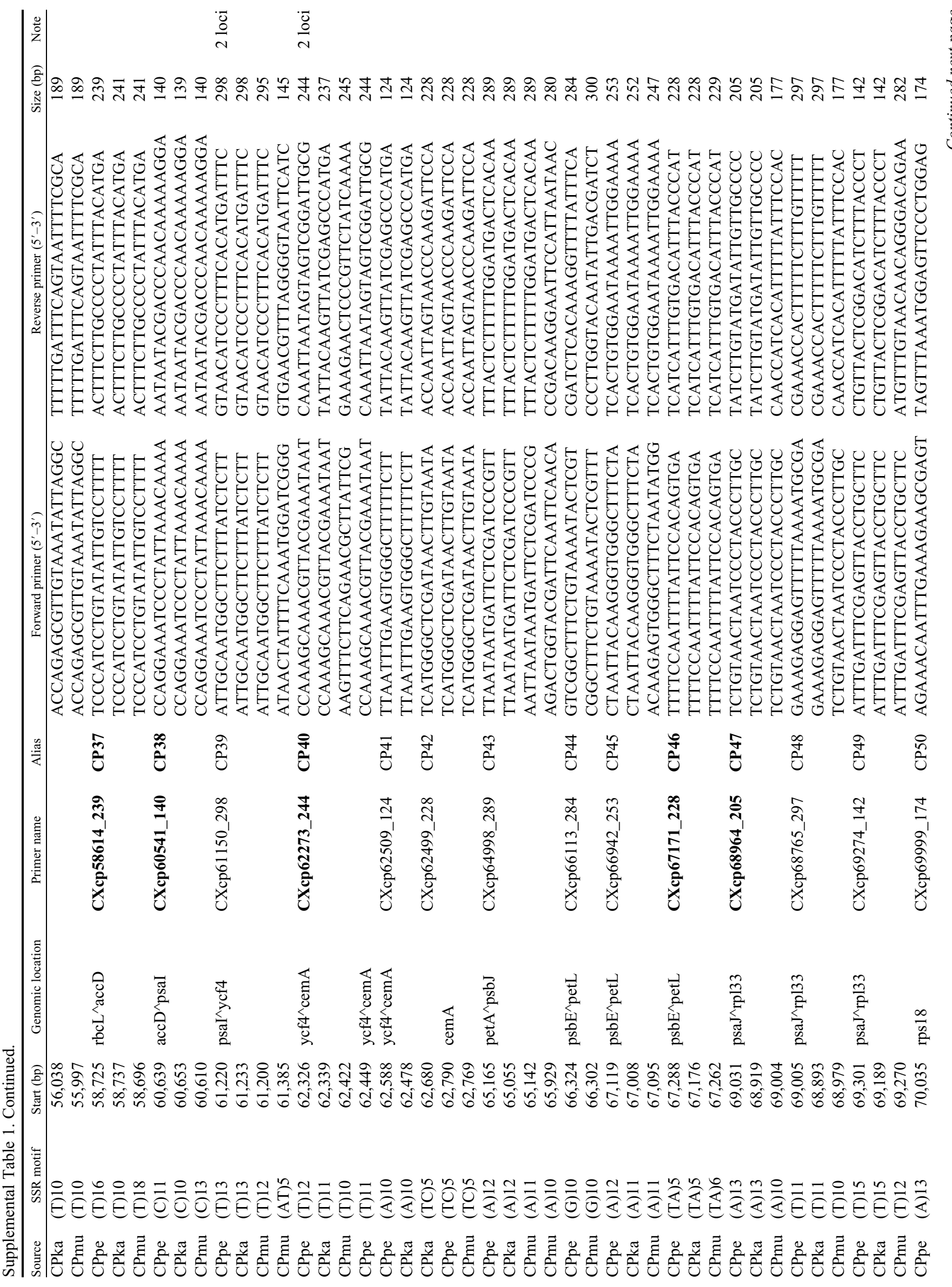




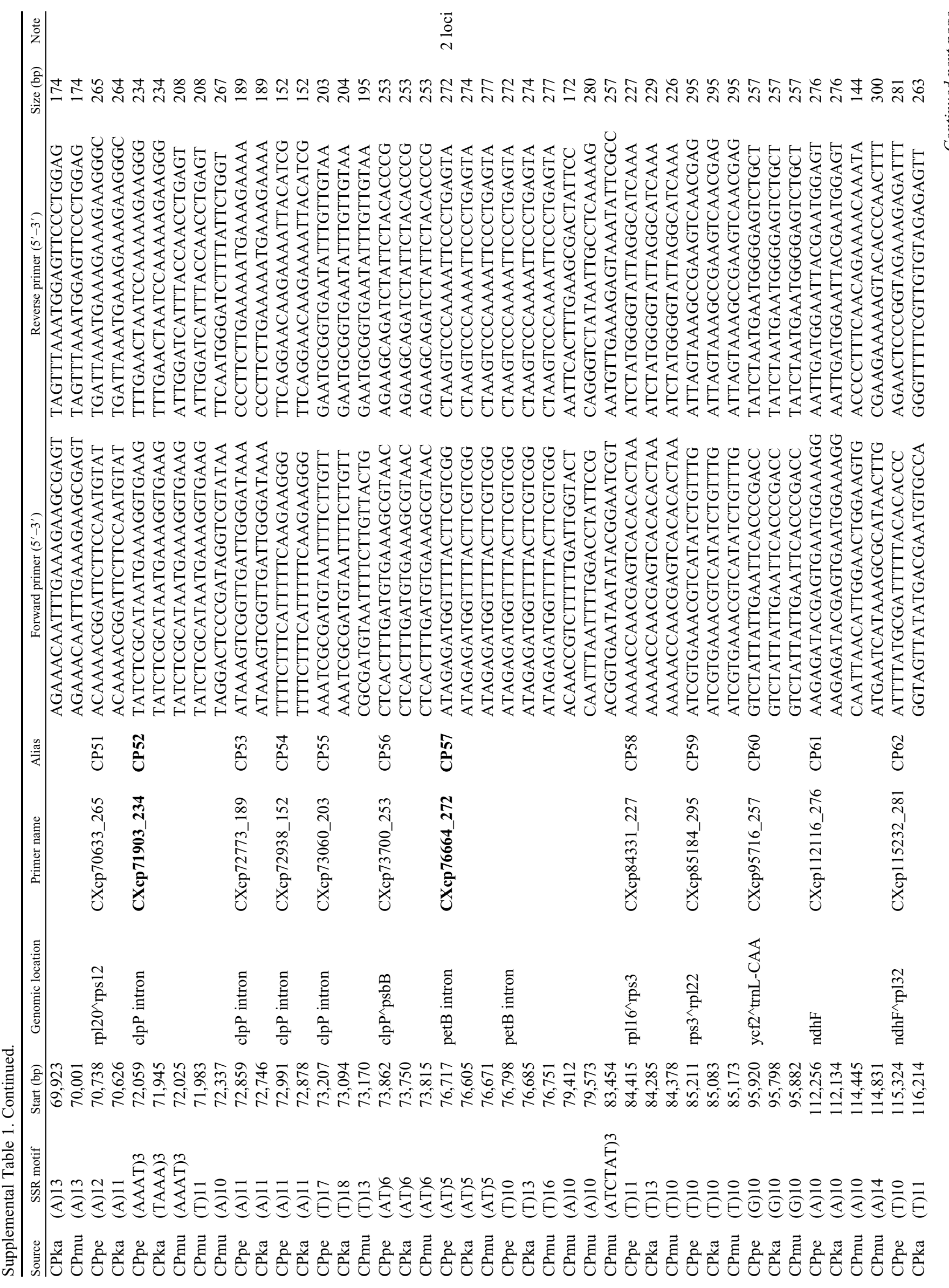




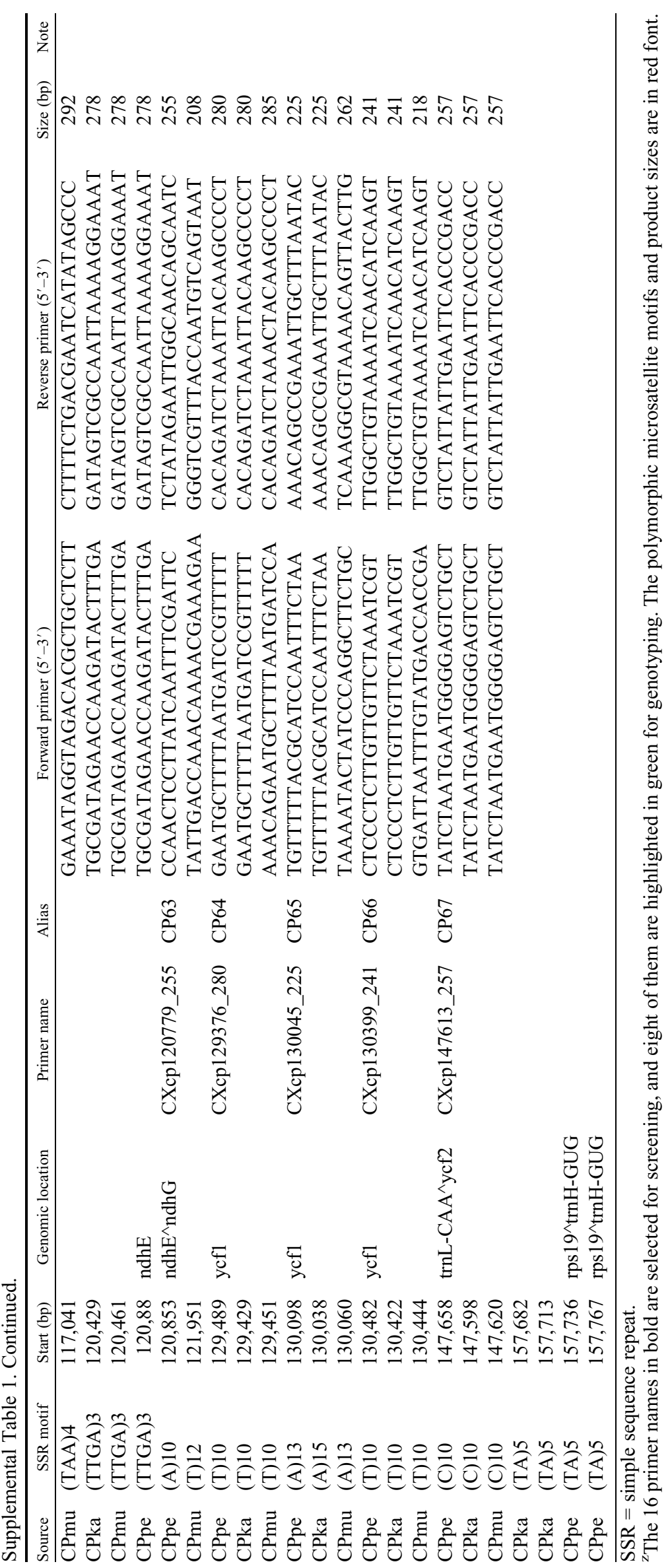


Supplemental Table 2. Peach cultivars and selections belonging to each maternal lineage group (MLG), along with first maternal ancestor and progenitor ancestor cultivar.

\begin{tabular}{|c|c|c|c|c|}
\hline$\overline{\mathrm{MLG}^{\mathrm{z}}}$ & Cultivar $^{y}$ & Selections (no.) & First maternal ancestor cultivar & Progenitor cultivar or type \\
\hline 1 & Arctic Belle & & Arctic Queen & JH Hale \\
\hline 1 & Arctic Gold & & Ruby Gold & JH Hale \\
\hline 1 & Arctic Sweet & & White Lady & JH Hale \\
\hline 1 & & 3 & $\begin{array}{l}\text { ARS-Kearneysville } \\
\text { selections }\end{array}$ & Unknown \\
\hline 1 & & 48 & ARS-Parlier selections & Unknown \\
\hline 1 & Autumnprince & 4 & O’Henry & JH Hale \\
\hline 1 & Belle of Georgia & & Chinese Cling & Chinese Cling \\
\hline 1 & Big Red $=$ CVN\#3 & & Unknown & Unknown \\
\hline 1 & Bill Gross Indian & & Unknown & Unknown \\
\hline 1 & & 8 & Biscoe & JH Hale \\
\hline 1 & Blazeprince & 10 & O’Henry & JH Hale \\
\hline 1 & Camden & & Hiley & JH Hale \\
\hline 1 & Carman & & Elberta & Chinese Cling \\
\hline 1 & Carolina Gold & & Biscoe & JH Hale \\
\hline 1 & Carolina Red & & Nectared 4 & JH Hale \\
\hline 1 & Carored & & Springbrite & JH Hale \\
\hline 1 & & 4 & Challenger & JH Hale \\
\hline 1 & Chinese Cling & & Unknown & Chinese Cling \\
\hline 1 & Clayton & & Pekin & JH Hale \\
\hline 1 & Compact Elberta & & Fay Elberta & Chinese Cling \\
\hline 1 & Contender & 5 & Winblo & JH Hale \\
\hline 1 & Coronet IR & & Halehaven & JH Hale \\
\hline 1 & Cresthaven & & Kalhaven & JH Hale \\
\hline 1 & Cresthaven IR & & Kalhaven & JH Hale \\
\hline 1 & Crimson Snow & 3 & Sungrand & JH Hale \\
\hline 1 & Dixiland & & Halehaven & JH Hale \\
\hline 1 & Durbin & & Flaming Gold & JH Hale \\
\hline 1 & Dwarf Elberta & & Elberta & Chinese Cling \\
\hline 1 & Eastern Glo & & Red Grand & JH Hale \\
\hline 1 & Elberta & & Chinese Cling & Chinese Cling \\
\hline 1 & Elberta & & Chinese Cling & Chinese Cling \\
\hline 1 & Empress & & Unknown & Unknown \\
\hline 1 & Fackler & & Unknown & Unknown \\
\hline 1 & Fairtime & & Rodeo & Late Crawford \\
\hline 1 & & 1 & Fire Pearl & Unknown \\
\hline 1 & Fireprince & 1 & Halberta Giant & JH Hale \\
\hline 1 & Flameprince & 29 & Summerset & Late Crawford \\
\hline 1 & Flameprince Ham & & Summerset & Late Crawford \\
\hline 1 & Flameprince Pearson & & Summerset & Late Crawford \\
\hline 1 & Flavorich & & May Grand & JH Hale \\
\hline 1 & & 11 & Flavortop & Late Crawford \\
\hline 1 & Flavrburst & & Blazeprince & JH Hale \\
\hline 1 & Flordadawn & & Flordagold & Unknown \\
\hline 1 & Flordaprince & & Southland & JH Hale \\
\hline 1 & Frank & & Elberta & Chinese Cling \\
\hline 1 & GaLa & & Harvester & JH Hale \\
\hline 1 & Glacier White & & Unknown & Unknown \\
\hline 1 & Greensboro & & Connett & Historic \\
\hline 1 & & 1 & Hakuho & Chinese Cling \\
\hline 1 & Hakuto & 1 & Chinese Cling & Chinese Cling \\
\hline 1 & Hiland & & Southland & JH Hale \\
\hline 1 & Hiley & & Belle of Georgia & Chinese Cling \\
\hline 1 & Honey Royale & & Unknown & Unknown \\
\hline 1 & J.H.Hale & & Elberta? & Chinese Cling \\
\hline 1 & Jefferson & & JH Hale & JH Hale \\
\hline 1 & Jerseyqueen & & Candoka & JH Hale \\
\hline
\end{tabular}

Continued next page 
Supplemental Table 2. Continued.

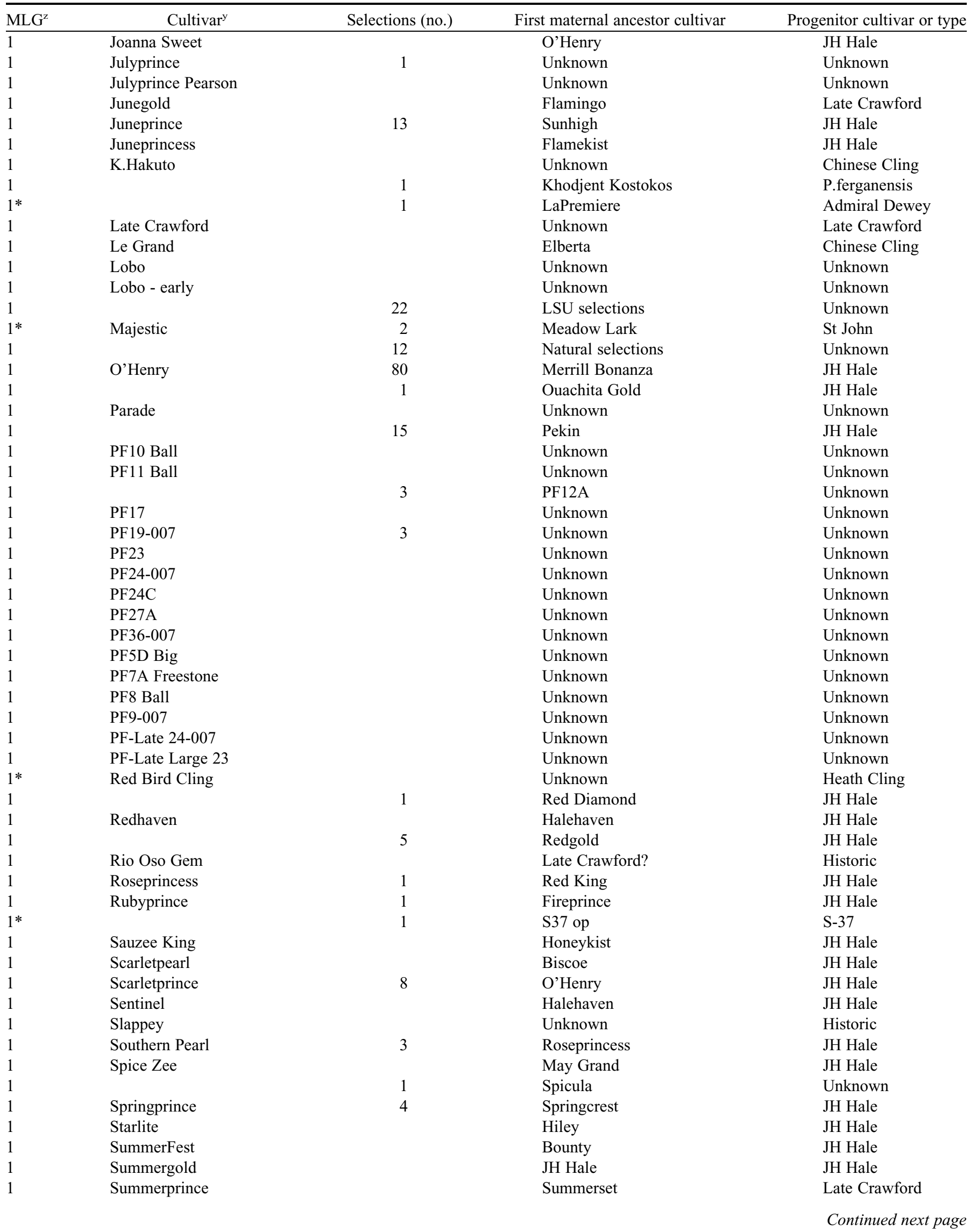


Supplemental Table 2. Continued.

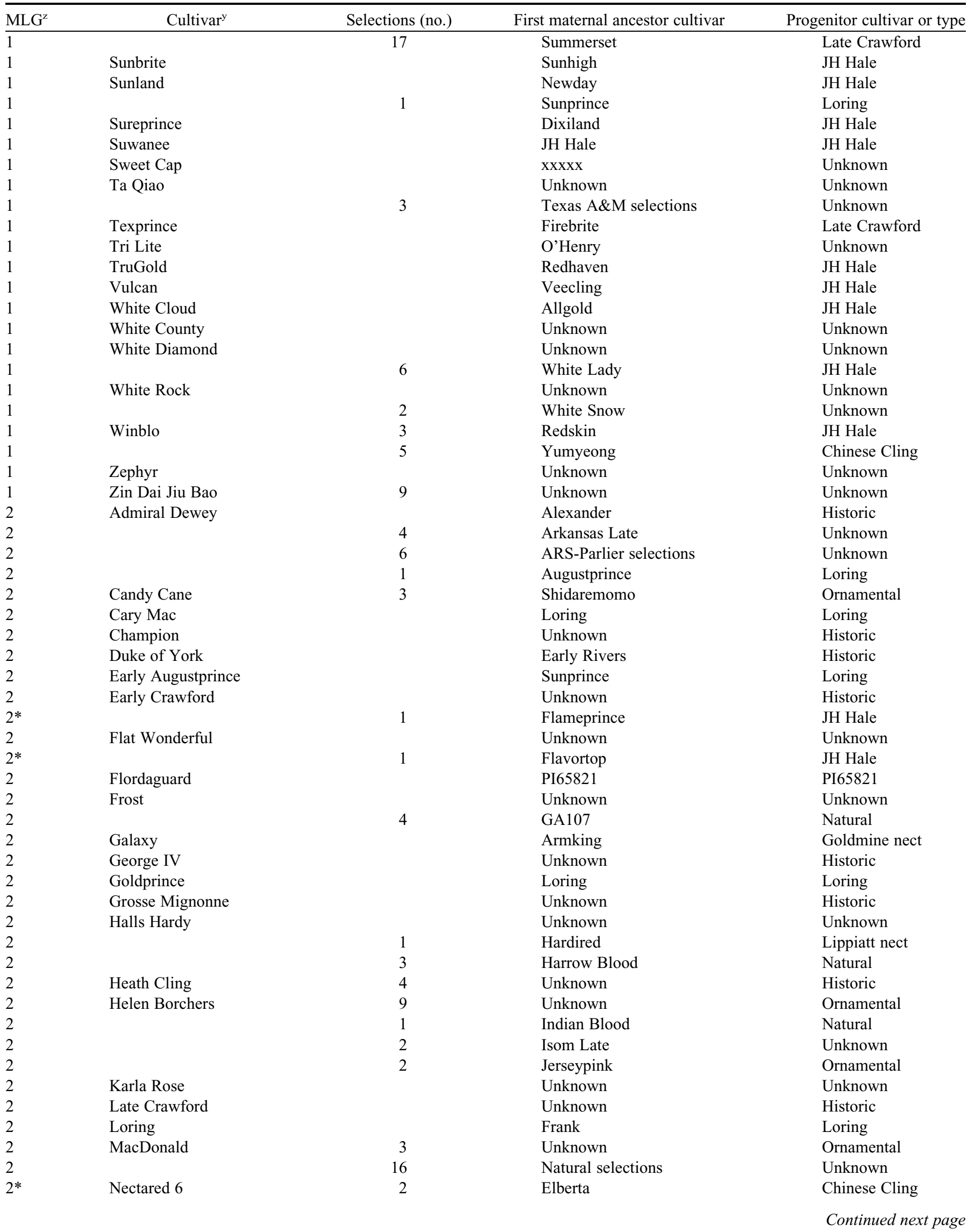


Supplemental Table 2. Continued.

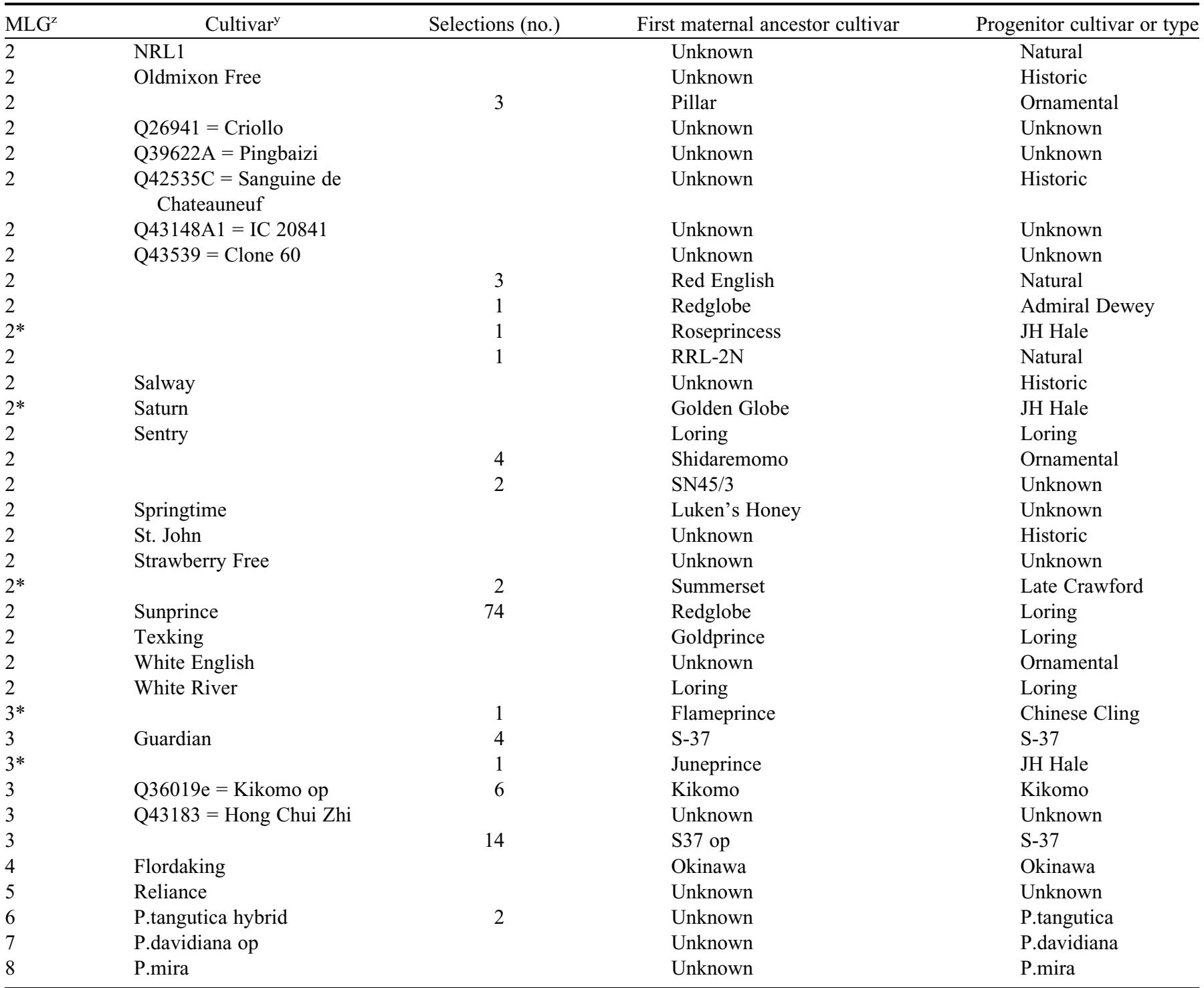

${ }^{\mathrm{z}}$ Asterisks indicate entries in a different group than expected based on known pedigree.

${ }^{\mathrm{y}}$ Where no cultivar is listed the parent was not tested, but the indicated number of selections were maternally derived from the first parent. 
Supplemental Table 3. Number of single nucleotide polymorphisms (SNPs) in the chloroplast amplicon sequences from the eight representative materials amplified by the eight primers.

\begin{tabular}{|c|c|c|c|c|c|c|c|c|}
\hline & $\mathrm{A} / \mathrm{G}$ & $\mathrm{C} / \mathrm{T}$ & $\mathrm{A} / \mathrm{C}$ & $\mathrm{G} / \mathrm{T}$ & $\mathrm{A} / \mathrm{T}$ & $\mathrm{C} / \mathrm{G}$ & Others & Total \\
\hline Primer name & \multicolumn{8}{|c|}{ SNPs (no.) } \\
\hline CXcp01488_232 & 1 & & & & 1 & & & 2 \\
\hline CXcp05368_299 & & & & & & & & 0 \\
\hline CXcp36648_290 & 10 & 3 & 2 & 2 & 6 & 1 & 1 & 25 \\
\hline CXcp58614_239 & 7 & 2 & 1 & 4 & 5 & & 2 & 21 \\
\hline CXcp60541_140 & & & & & & & & 0 \\
\hline CXcp62273_244 & 1 & & & & 1 & & & 2 \\
\hline CXcp68964_205 & 3 & 1 & 1 & 3 & 4 & 1 & & 13 \\
\hline CXcp76664_272 & 1 & & & 1 & 1 & & & 3 \\
\hline
\end{tabular}

